\section{Mortalidade relacionada à asma no Município do Rio de Janeiro, Brasil, no período de 2000-2009: análise de causas múltiplas}

\author{
Asthma-related mortality in the city of Rio de \\ Janeiro, Brazil, 2000-2009: a multicausal analysis
Mortalidad relacionada con el asma en el Municipio de Río de Janeiro, Brasil, durante el período de 2000-2009: análisis de causas múltiples

\section{${ }^{1}$ Universidade Federal do Estado do Rio de Janeiro, Rio de Janeiro, Brasil. 2 Universidade do Estado do Rio de Janeiro, Rio de Janeiro, Brasil. \\ Correspondência E. M. Silva Universidade Federal do Estado do Rio de Janeiro. Rua Assis Brasil 111, apto. 1101, Rio de Janeiro, RJ 22030-010, Brasil. elianemir@live.com}

\begin{abstract}
The study of multiple causes of death helps reveal the true extent of mortality statistics. The aim of this study was to assess trends in asthma mortality rates in the city of Rio de Janeiro, Brazil, from 2000 to 2009, based on data from the Mortality Information System among individuals one year or older, in which asthma was mentioned on any line or in any part of the death certificate. Linear regression was used for data analysis. The historical series showed a downward trend in the standardized mortality rate related to asthma as the underlying or associated cause of death, with a reduction in males and stability in females. When asthma was the underlying cause, the most common associated causes were diseases of the respiratory system. Asthma-related mortality was underestimated when considered only as the underlying cause, which could be avoided by the use of a multicausal methodology in asthma mortality statistics.
\end{abstract}

Asthma; Multiple Causes of Death; Mortality
Eliane Miranda da Silva 1 Gulnar Azevedo e Silva 2

\section{Resumo}

O estudo das causas múltiplas de óbitos permite conhecer a extensão real das estatísticas de mortalidade. O objetivo desta pesquisa foi avaliar a tendência das taxas de mortalidade relacionada à asma, no Município do Rio de Janeiro, Brasil, no período de 2000-2009, com dados obtidos no Sistema de Informações de Mortalidade (SIM), de indivíduos com um ano ou mais de idade, em que a asma foi mencionada em qualquer $l i-$ nha ou parte do atestado médico da declaração de óbito. Para análise de dados foi utilizada a técnica de regressão linear. A série histórica mostrou tendência ao declínio nas taxas de mortalidade padronizadas relacionada à asma como causa básica e múltipla, com redução entre os homens e estabilidade entre as mulheres. Quando a asma foi a causa básica, as causas associadas mais frequentes foram doenças do aparelho respiratório. A mortalidade relacionada à asma foi subestimada quando considerada apenas como causa básica, o que poderia ser evitado com a utilização da metodologia de causas múltiplas nas estatísticas de mortalidade por asma.

Asma; Causas Múltiplas de Morte; Mortalidade 


\section{Introdução}

A asma é uma doença pulmonar inflamatória de distribuição mundial. A partir dos anos 60, observou-se uma tendência crescente na prevalência da asma em todos os continentes, particularmente entre as décadas de 80 e 90, principalmente em regiões desenvolvidas. Uma grande variação na prevalência da asma, de $1 \%$ a $18 \%$, é observada nos diferentes países do mundo 1 . Essa variabilidade é proporcional à urbanização e à adoção do estilo de vida ocidental. $\mathrm{O}$ aumento da prevalência em regiões em desenvolvimento, como África, América Latina e partes da Ásia, demonstra que a diferença entre os países está diminuindo. Estima-se que em 2025 esses números serão acrescidos de mais 100 milhões de pessoas 1 .

Apesar de ser uma doença de alta prevalência, o óbito por asma é um evento raro, porém de significância epidemiológica. Os registros oficiais de óbitos são a principal fonte de dados utilizados nos estudos de mortalidade, constituindo a melhor informação para quantificar mortalidade, conhecer o perfil epidemiológico de uma região e planejar programas de prevenção em saúde pública.

Em 2009, Wijesinghe et al. 2 avaliaram a mortalidade por asma em 20 países da Europa, Ásia e Estados Unidos, no período de 1960-2005, em indivíduos de 5-34 anos. Observaram que, a partir de 1985/1986, ocorreu um declínio nas taxas de mortalidade, alcançando um índice de 0,23 por 100 mil habitantes em 2004 e 2005. O momento do declínio dessas taxas variou entre os diversos países 2. No Brasil, no período de 1980-1998, Campos ${ }^{3}$ observou um declínio na taxa de mortalidade, que passou de 1,93/100 mil em 1980 para 1,41/100 mil em 1998.

No Brasil, existem poucos estudos descritivos sobre a prevalência e mortalidade por asma, embora a prevalência de asma ativa seja elevada, segundo dados do estudo ISAAC (International Study of Asthma and Allergies in Childhood $24,3 \%$ na idade de $6-7$ anos e $19 \%$ entre os adolescentes de 13-14 anos) 4 .

Dentre os diversos fatores de risco associados à morte por asma, destaca-se o tabagismo, cuja prevalência entre os indivíduos com a doença é de $20-35 \%$, com predomínio entre as mulheres, como mostraram Silverman et al. 5 em um estudo multicêntrico, nos Estados Unidos e Canadá. Analisaram 2.000 pacientes atendidos no setor de emergência para tratamento de crise de asma e verificaram que $35 \%$ destes eram fumantes ${ }^{5}$. A exposição à fumaça do cigarro produz alterações fisiopatológicas nas vias aéreas e interfere na resposta terapêutica em pacientes com asma não controlada, mesmo após parar de fumar 6 .
No Brasil, em 2010, a frequência de fumantes foi de $15,1 \%$, sendo $17,9 \%$ no sexo masculino e $12,7 \%$ no feminino. Entre as mulheres, a prevalência do tabagismo apresentou tendência de aumento com a idade até os 54 anos (de 12,4\% na faixa etária de $18-24$ anos para $16,4 \%$ na faixa etária de 45-54) e sofreu queda nas faixas etárias subsequentes, chegando a $6,5 \%$ entre aquelas com 65 ou mais anos de idade 7 . Em vários países, incluindo o Brasil, medidas de prevenção e controle do tabagismo têm resultado na queda significativa da prevalência de fumantes nas últimas décadas.

A asma é uma doença que não tem cura, mas é de morte potencialmente evitável. O impacto do uso do corticoide na redução da morbimortalidade da asma foi evidenciado em vários estudos $8,9,10,11$. A prevalência, a gravidade do quadro e a conduta médica adotada na comunidade em estudo vão influenciar as taxas de hospitalização por asma ${ }^{12}$. A implementação de programas de combate à asma focados na atenção primária, com a participação de equipe multidisciplinar e fornecimento gratuito de medicação, tem se tornado cada vez mais importante. No Brasil, programas de controle da asma têm sido implantados em diversos municípios, os quais têm contribuído com significativa redução na morbidade por esta doença 13,14. Cerci Neto et al. 15 analisaram 17 programas brasileiros e concluíram que já existem experiências acumuladas suficientes para a criação de um programa nacional de capacitação profissional em asma. No Município do Rio de Janeiro, o RespiraRio, implementado em 2004, vem melhorando a qualidade da assistência aos pacientes com asma grave, com consequente redução da hospitalização por esta doença no município 16.

Tradicionalmente, as estatísticas de mortalidade baseiam-se apenas em uma causa, denominada causa básica de morte. As mortes relacionadas às doenças crônicas raramente resultam de um único estado mórbido e nem sempre podem ser caracterizadas adequadamente por meio da causa básica. Nesse cenário, em que uma única causa não abrange todas as circunstâncias do evento mórbido que culminou na morte, o estudo das causas múltiplas de morte pode analisar, com maior precisão, as doenças e complicações presentes no momento do óbito.

Na França, no período de 2000-2005, a asma foi mencionada como causa básica em $44 \%$ dos óbitos. O estudo por causas múltiplas possibilitou o resgate de $118 \%$ de óbitos por asma, passando de 8.284 para 18.936 mortes 17 . No Brasil, Santo 18 mostrou que, no ano 2000, as taxas de mortalidade aumentaram aproximadamente $50 \%$, quando todas as menções da Declaração de Óbito (DO) 
foram analisadas. Esse mesmo autor verificou que a subestimação entre os coeficientes brutos de mortalidade nos anos 1999 e 2000 foi, respectivamente, no Rio Grande do Sul de $62 \%$ e $75,4 \%$, e em São Paulo de 54,7\% e 53,1\% 19.

Considerando que a análise da causa básica de morte não possibilita a compreensão real da magnitude do quadro de mortalidade por asma, a análise incluindo causas múltiplas pode prover conhecimentos acerca da coexistência de doenças que levaram ao óbito, e que poderão ser utilizados como subsídios para o planejamento e a avaliação da qualidade das ações de prevenção e controle. $\mathrm{O}$ objetivo deste estudo foi analisar a tendência da mortalidade por asma, considerando-a como causa múltipla de óbito, no Município do Rio de Janeiro, no período de 2000-2009.

\section{Métodos}

Os dados de óbitos foram obtidos com base nas informações registradas no Sistema de Informações sobre Mortalidade (SIM. Departamento de Informática do SUS. http://www.datasus.gov.br, acessado em 05/Out/2011), no período de 20002009, nas DO de residentes no Município do Rio de Janeiro, Brasil, em indivíduos com idade $\geq 1$ ano.

Foram consideradas todas as DO que mencionaram a asma em qualquer linha nas partes I e II, sob os códigos J45 (Asma) e J46 (Estado de mal asmático), referentes à 10a revisão da Classificação Internacional de Doenças (CID-10).

A terminologia adotada para as causas de mortes foi a seguinte: para causa básica, foi utilizada a definição da Organização Mundial da Saúde (CID-10): causas intervenientes são todas as complicações ou consequências da causa básica, declaradas nas linhas da Parte I do atestado de óbito, acima da causa básica; causas contribuintes são as assinaladas na Parte II do atestado de óbito. As causas associadas são todas as causas de morte informadas que não constituem a causa básica. Denomina-se causa múltipla o conjunto causa básica mais causa associada. As causas consequenciais e contribuintes não foram avaliadas individualmente, sendo analisadas sob a denominação de causas associadas de morte. As causas declaradas como mal definidas (classificadas no capítulo XVIII - R00 a R99 - da CID-10) e aquelas caracterizadas pela OMS como modos de morrer, como a insuficiência respiratória, também foram consideradas na análise de causas de morte.

Foi calculada a relação entre o total de vezes em que a asma foi mencionada em qualquer linha da DO pelo total de menções como causa básica para cada ano estudado. Esse indicador é denominado razão de menção de causa básica.

Tendo em vista que a contribuição das causas mal definidas no período estudado foi inferior a $10 \%$ e que os registros de óbitos por asma têm sensibilidade de $42 \%$ e especificidade de $99 \% 20$, optou-se por não corrigir as taxas de mortalidade por asma segundo a redistribuição dos óbitos por causas mal definidas.

O número de habitantes para o cálculo das taxas de mortalidade no Município do Rio de Janeiro por asma foi obtido nos censos demográficos do Instituto Brasileiro de Geografia e Estatística (IBGE. http://www.ibge.gov.br, acessado em 12/Jan/2012). Para os anos intercensitários, os denominadores populacionais foram estimados por meio de interpolação linear com base nos dados censitários.

As taxas de mortalidade específicas foram calculadas para as seguintes faixas etárias: 1-4 anos, 5-9, 10-14, 15-19, 20-34, 35-59, 60 anos e mais, considerando-se a asma como causa básica e como causa múltipla, segundo gênero para cada ano do período. As taxas foram padronizadas por idade, conforme a técnica de padronização pelo método direto, sendo adotada, como população padrão, aquela dos residentes no Município do Rio de Janeiro (Censo Demográfico de 2010. http:// www.ibge.gov.br, acessado em 12/Jan/2012).

Foi realizada análise de tendência das taxas de mortalidade por asma, padronizadas por idade, segundo causas básicas e múltiplas, utilizandose análise de regressão linear simples e regressão linear segmentada (piecewise linear regression) 21. Usou-se como variável dependente a taxa de mortalidade padronizada por asma, e como variável independente os anos calendários do estudo. Essa última modelagem permite estimar separadamente a performance em cada segmento de análise, assumindo que, em cada segmento, as retas têm diferentes interceptos e inclinações. $\mathrm{O}$ ponto de transição testado foi o ano de 2004, com base na visualização gráfica. As conclusões foram tomadas no nível de 95\% de significância.

Nos casos em que uma causa foi citada mais de uma vez dentro de uma categoria, agrupamento ou capítulo da CID-10 na mesma DO, considerou-se apenas uma vez a informação referente a esta causa. Assim, a duplicação e multiplicação de causas de óbitos foram eliminadas.

Toda a análise dos dados foi realizada no programa R (The R Project for Statistical Computing; http://www.r-project.org). 


\section{Resultados}

No período de 2000-2009, no Município do Rio de Janeiro, foram registrados 512.082 óbitos, dos quais em 1.090 a asma foi classificada como a causa básica. Foi mencionada como causa associada em outras 531 DO, totalizando 1.621 óbitos com menção de asma. A recuperação dos óbitos por asma como causa associada elevou em $48,7 \%$ o total de mortes por esta doença, em relação à sua menção como causa básica. A mortalidade proporcional por asma, considerando as causas múltiplas no período, foi de $0,35 \%$, a razão ho$\mathrm{mem} / \mathrm{mulher}$ foi, em média, 1:2,3, e a maior parte dos óbitos (61\%) ocorreu entre os indivíduos de 60 anos e mais. O número médio de diagnósticos informados nas DO como causas múltiplas, no período de 2000-2009, foi igual a 3,4. Apenas $4 \%$ dos óbitos apresentaram uma única causa registrada na DO. Em 73,3\% dos óbitos foram registradas três causas ou mais. A média da razão de menção de causa básica nos dez anos de estudo variou entre 1,35 e 1,65.

As taxas de mortalidade padronizadas por asma segundo causas básicas, associadas e múltiplas no Município do Rio de Janeiro, para o período estudado, são apresentadas na Tabela 1. Em 2000, a taxa de mortalidade padronizada por asma como causa básica era de 2,22/100 mil habitantes e passou para 1,72/100 mil habitantes em 2009, correspondendo a um decréscimo de $22,5 \%$.

Considerando todas as menções de asma, observou-se uma redução na taxa de mortalidade padronizada igual a $18,3 \%$, que em 2000 era $3,45 / 100$ mil habitantes passando para 2,82/100 mil habitantes em 2009. Esse declínio na taxa de mortalidade por causas múltiplas foi decorrente, principalmente, da redução de $22,5 \%$ na taxa de mortalidade por causa básica, contra uma pequena variação de $10,6 \%$ nas taxas de mortalidade por causas associadas (Tabela 1).
A Figura 1 mostra a tendência das taxas de mortalidade total e por gênero, segundo causas básicas e múltiplas, entre 2000 e 2009. No conjunto, a tendência linear foi decrescente $(\beta=-0,06, p=0,017$ e $\beta=-0,11, p=0,005$, respectivamente). Estratificando por gênero, evidenciou-se um declínio nas taxas padronizadas de asma como causa básica entre os homens de $62,7 \%$, passando de $1,58 / 100$ mil em 2000 para $0,59 / 100$ mil em 2009 ( $\beta=-0,08$, $\mathrm{p}=0,007$ ); como causas múltiplas as taxas diminuíram de 2,49/100 mil em 2000 para 1,11/100 mil em 2009, correspondendo a uma queda de $55,4 \%(\beta=-0,14, p<0,00001)$ (Figura 2). Entre as mulheres, as taxas de mortalidade passaram de 2,79/100 mil em 2000 para 2,72/100 mil em 2009 como causa básica, configurando uma redução de $2,5 \%$, e de 4,29/100 mil em 2000 para $4,32 / 100$ mil em 2009, o equivalente à elevação de $0,7 \%$ nas taxas como causas múltiplas (Figura 3). A tendência das taxas como causa básica, entre as mulheres, foi analisada em dois períodos, de 2000-2004 e 2004-2009, e não apresentou significância estatística (2000-2004: $\beta=-0,16, p=0,131 ; 2004-2009$ : $\beta=0,04, p=0,630$ ).

A análise por faixa etária apresentou significância estatística apenas para os homens de 2034 anos $(\beta=-0,04, p=0,027)$ e 60 anos e mais $(\beta=$ $-0,29, \mathrm{p}=0,015)$ para causa básica, e de 60 anos e mais $(\beta=-0,58, p=0,001)$ para causa múltipla.

Na Tabela 2, são apresentadas as principais causas associadas mencionadas nas DO em que a asma foi classificada como causa básica, no primeiro (2000-2002) e último triênios (2007-2009) da série, segundo gênero e faixa etária. Os resultados foram apresentados em porcentuais calculados em relação ao número de óbitos como causas básicas. Entre crianças e adolescentes de 1-19 anos, as causas mais frequentes foram insuficiência respiratória e pneumonia; nos grupos etários entre 20-34 anos, apenas a insuficiência respiratória se destaca; entre 35 e 59, além da insuficiência respiratória volta a aparecer pneumonia e,

Tabela 1

Taxa de mortalidade * relacionada à asma. Município do Rio de Janeiro, Brasil, 2000 e 2009.

\begin{tabular}{|c|c|c|c|c|c|c|c|c|c|}
\hline & \multicolumn{3}{|c|}{ Total } & \multicolumn{3}{|c|}{2000} & \multicolumn{3}{|c|}{2009} \\
\hline & n (\%) & $\begin{array}{l}\text { Taxa } \\
\text { bruta }\end{array}$ & $\begin{array}{c}\text { Taxa } \\
\text { padronizada }\end{array}$ & n (\%) & $\begin{array}{c}\text { Taxa } \\
\text { bruta }\end{array}$ & $\begin{array}{c}\text { Taxa } \\
\text { padronizada }\end{array}$ & n (\%) & $\begin{array}{l}\text { Taxa } \\
\text { bruta }\end{array}$ & $\begin{array}{c}\text { Taxa } \\
\text { padronizada }\end{array}$ \\
\hline Causa básica & $1.090(67,2)$ & 1,82 & 1,95 & $112(66,3)$ & 1,94 & 2,22 & $105(61,0)$ & 1,69 & 1,72 \\
\hline Causa associada & $531(32,8)$ & 0,89 & 0,97 & $57(33,7)$ & 0,99 & 1,23 & $67(39,0)$ & 0,91 & 1,10 \\
\hline Causa múltipla & $1.621(100,0)$ & 2,71 & 2,92 & $16(100,0)$ & 2,93 & 3,45 & $172(100,0)$ & 2,77 & 2,82 \\
\hline
\end{tabular}

* Por 100 mil habitantes. Padronizada por idade pela população do Município do Rio de Janeiro, Censo Demográfico 2010.

Fonte: Departamento de Informática do SUS (Sistema de Informações sobre Mortalidade. http://www.datasus.gov.br, acessado em 05/Out/2011). 


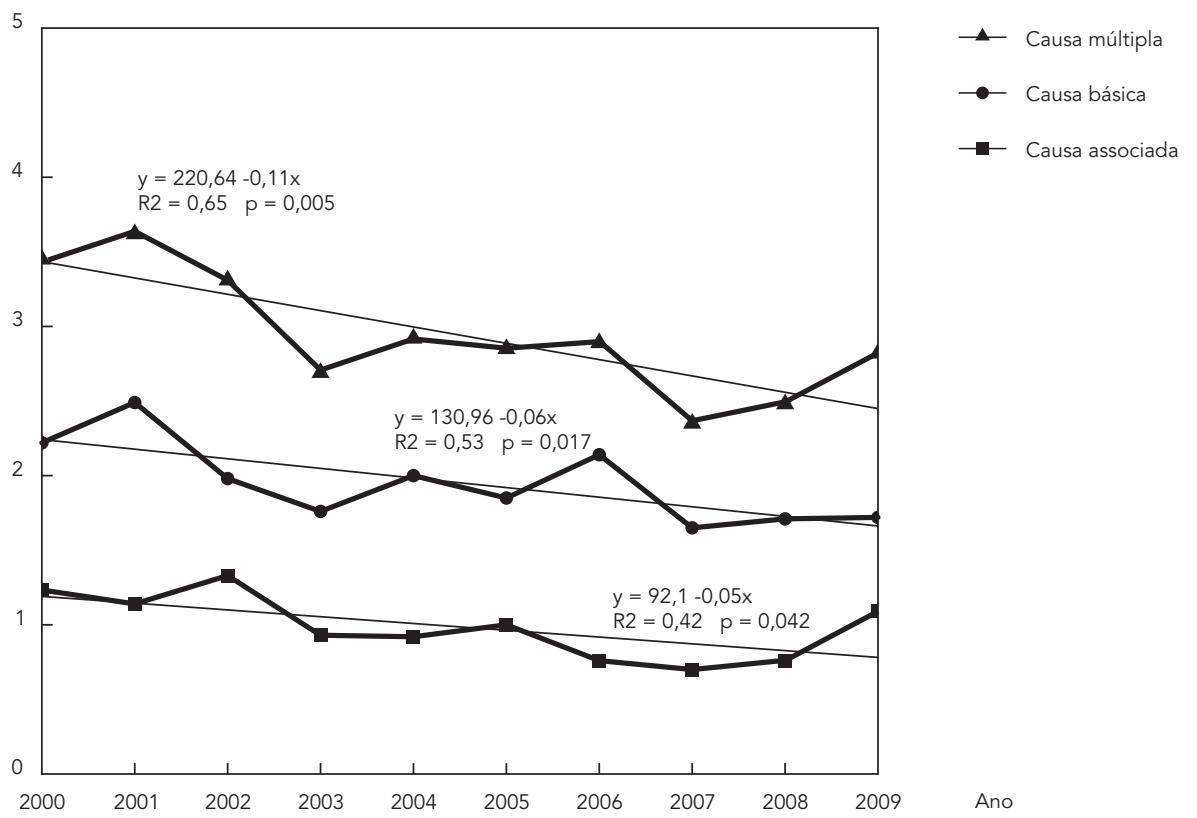

* Por 100 mil habitantes padronizada por idade pela população do Município do Rio de Janeiro, Censo Demográfico 2010. Fonte: Departamento de Informática do SUS (Sistema de Informações sobre Mortalidade. http://www.datasus.gov.br, acessado em 05/Out/2011).

a partir dos 60 anos, os diagnósticos mais citados são: insuficiência respiratória, doença pulmonar obstrutiva crônica (DPOC) e pneumonia, destacando-se também no último triênio a doença hipertensiva.

Na Tabela 3, observam-se as causas básicas classificadas no SIM, quando a asma foi causa associada na DO, distribuídas segundo gênero e faixa etária, nos mesmos triênios. Entre as crianças de 1-4 anos, todos os óbitos por asma foram registrados na causa básica, com exceção de apenas um. Não ocorreu nenhum óbito entre homens de 5-9 anos no triênio de 2007-2009. Entre crianças e adultos nas idades de 5-34 anos, os óbitos que foram recuperados tendo a asma como causa associada tiveram, na sua maioria, como causas básicas, as doenças do aparelho respiratório. Para os indivíduos de 35 a 59 anos, as causas básicas mais frequentes foram doença hipertensiva e doença cardíaca isquêmica. Para os idosos (60 anos e mais), as causas básicas mais frequentemente relatadas foram doença hipertensiva, DPOC e doença isquêmica.

\section{Discussão}

Nesta pesquisa, após a inclusão de causas múltiplas, a magnitude da mortalidade por asma aumentou em $48,7 \%$. A análise considerando todas as causas que aparecem no atestado de óbito permitiu uma melhor aproximação da asma entre as causas que levam à morte. Esse resultado foi semelhante ao descrito por Santo 18 , que encontrou uma diferença de $49,7 \%$ ao analisar os óbitos por asma em sete estados do Brasil em 2000, com uma variação de 35,4\% no Ceará a 76,1\% no Rio Grande do Sul.

Para algumas doenças, apenas com a utilização da metodologia de causas múltiplas de morte é possível conhecer a real frequência de tais patologias como causa de óbito. Mesmo que sejam assinaladas pelo médico como a causa que deu início à sequência do processo mórbido, após a aplicação das regras de codificação elas muitas vezes deixam de aparecer como causa básica.

Ao se avaliar a razão de menção de causa básica, verificou-se que a asma foi mencionada uma vez e meia em relação à sua classificação 


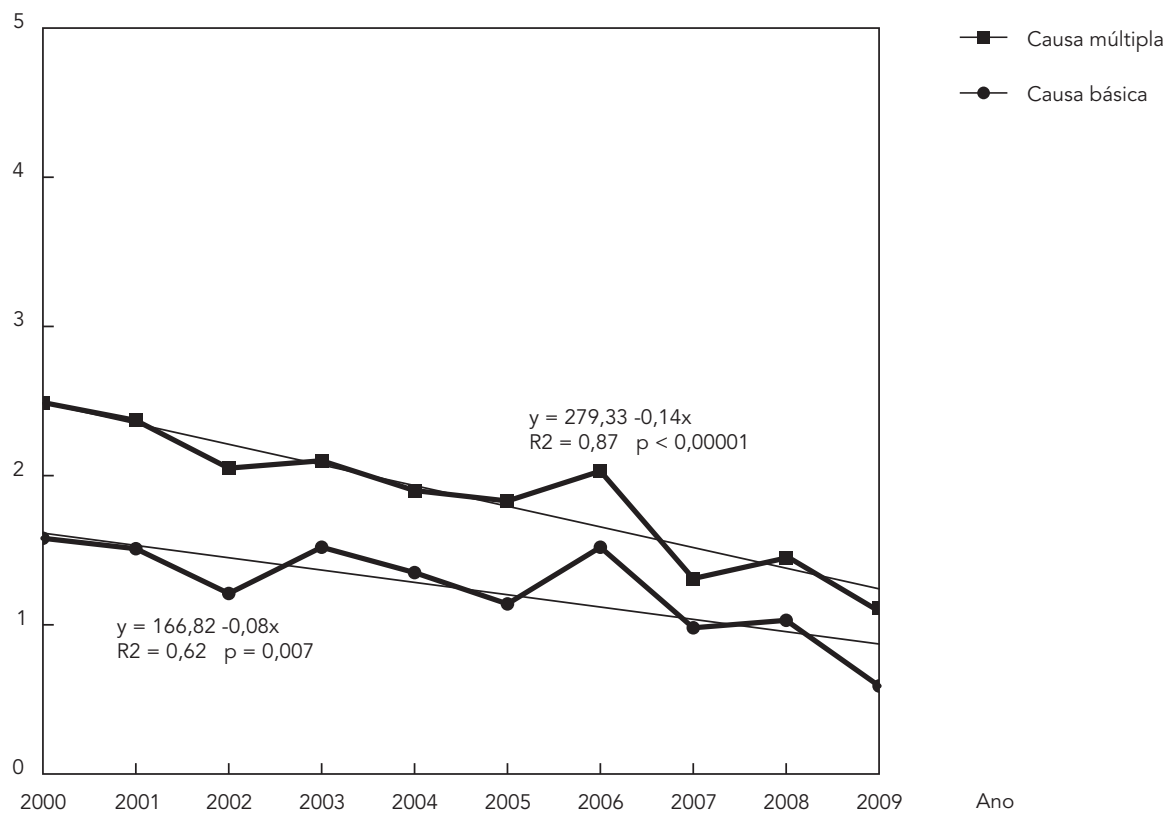

* Por 100 mil habitantes padronizada por idade pela população do Município do Rio de Janeiro, Censo Demográfico 2010. Fonte: Departamento de Informática do SUS (Sistema de Informações sobre Mortalidade. http://www.datasus.gov.br, acessado em 05/Out/2011).

como causa básica; mesma relação verificada em outros estados do país 18. Esse indicador reflete a influência de determinada patologia na produção de uma sequência de eventos que levam à causa terminal de morte.

A magnitude das taxas de mortalidade por asma entre mulheres foi sempre maior quando comparada à dos homens, diferentemente de quando se avalia a mortalidade geral no Município do Rio de Janeiro, onde as taxas entre os homens são maiores do que entre as mulheres. O predomínio da mortalidade por asma entre as mulheres também foi observado por outros autores. No National Asthma Survey nos Estados Unidos, publicado em 2007, observou-se um predomínio dos óbitos por asma em mulheres durante todo o período estudado, de 1980-2004 ${ }^{22}$. No Brasil, Oliveira et al. 23 em estudo realizado na cidade de Cascavel, Paraná, no período de 2005 2009, observaram que nos anos de 2005 e 2008 a taxa de mortalidade predominou entre homens, ao passo que, em 2006 e 2007, ocorreu o inverso, e em 2009 não houve diferença entre os sexos.

Diversas hipóteses têm sido apresentadas para esclarecer tais diferenças entre homens e mu- lheres, como: maior hiper-responsividade brônquica das mulheres 24 , uso incorreto da técnica de utilização dos dispositivos de aplicação das medicações inalatórias observado nas mulheres 25 , tabagismo 26, maior frequência de asma entre as mulheres, além do aumento da prevalência da asma na mulher a partir da puberdade 26 .

A tendência decrescente da mortalidade por asma encontrada neste estudo segue na mesma direção do observado em outros países, onde se verificou declínio na mortalidade por asma desde o final da década de 90 . Na França, no período de 2000 a 2005, as taxas de mortalidade por asma reduziram em $32 \%$, e a asma foi listada como causa básica de óbito em $44 \%$ das DO que fizeram menção à doença ${ }^{17}$. Essa tendência ao declínio também foi observada em um estudo nos Estados Unidos, no período de 1990-2001, que evidenciou uma queda de aproximadamente $20 \%$ e registro de morte por asma como causa básica em $45 \%$ do total de menções de asma 27.

Um ponto a ser destacado é a diferença na tendência da mortalidade entre os sexos na série estudada. Dentre as mulheres, observou-se uma tendência à estabilidade das taxas, enquanto en- 


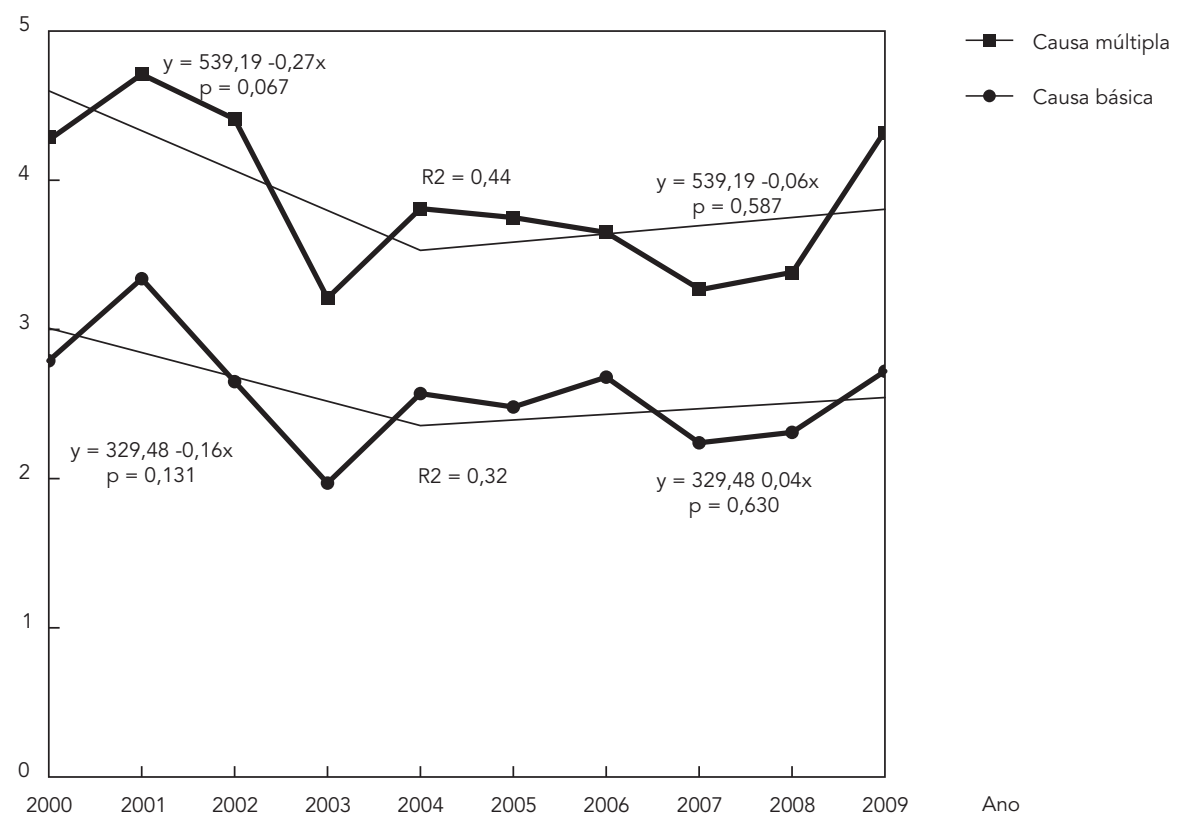

* Por 100 mil habitantes padronizada por idade pela população do Município do Rio de Janeiro, Censo Demográfico 2010. Fonte: Departamento de Informática do SUS (Sistema de Informações sobre Mortalidade. http://www.datasus.gov.br, acessado em 05/Out/2011).

tre os homens o declínio nas taxas ficou evidente em todo o período. Essa diferença acentuada na tendência entre os sexos tambémfoiverificadapor Fuhrman et al. 17. Eles encontraram uma redução de $3,4 \%$ e $8,7 \%$ em mulheres e homens, respectivamente, no período de 2000 a 2005 17. Também no Brasil, Chatkin et al. 28, em Pelotas, Rio Grande do Sul, no período de 1981-2003, mostraram uma taxa de mortalidade por asma $10 \%$ maior entre as mulheres do que entre os homens, com declínio maior entre os homens e estabilização ou até mesmo uma discreta elevação entre as mulheres 28 .

Uma explicação para a diferença acentuada entre os sexos na tendência da mortalidade por asma pode ser a queda mais acentuada na prevalência do tabagismo entre os homens ${ }^{7}$. Silva et al. 29 evidenciaram tendência ao declínio do tabagismo entre os homens e estabilidade entre as mulheres. É possível que a velocidade mais lenta na redução do consumo de tabaco entre as mulheres justifique uma maior prevalência da asma neste grupo e maior risco de hospitalização e morte.

É importante ressaltar que a grande queda nos óbitos por asma, observada entre os homens, foi influenciada pelo declínio entre os mais idosos, que teve uma tendência decrescente de $0,29 \%(p=0,015)$ ao ano. Além da influência do tabagismo, outra possibilidade é a presença de comorbidades nessa faixa etária, na qual a asma frequentemente é confundida com outras doenças, principalmente a DPOC. A dificuldade na distinção entre asma e DPOC favorece a baixa acurácia dos dados e a subestima no diagnóstico da asma, principalmente nos indivíduos com 60 anos e mais. Os estudos de mortalidade por asma têm maior fidedignidade na faixa etária de 5-34 anos, fase em que as comorbidades são menos frequentes 30 .

Outros fatores, além da queda do tabagismo, podem ter contribuído para a redução das taxas de mortalidade por asma a partir do final da década de 90, como a padronização do uso dos corticoides inalatórios apresentada nos manuais de procedimentos, os avanços no diagnóstico e o maior conhecimento sobre a doença nos últimos anos.

A educação dos pacientes e de seus familiares em relação ao manuseio terapêutico da doença e do ambiente, e a maior cobertura vacinal contra 
Tabela 2

Causas associadas referidas nos óbitos por asma como causa básica, segundo gênero e faixa etária. Município do Rio de Janeiro, Brasil, $2000-2002$ e 2007-2009.

\begin{tabular}{|c|c|c|c|c|c|c|c|c|c|c|c|c|c|c|}
\hline \multirow{3}{*}{$\begin{array}{l}\text { Causas associadas à } \\
\text { CID-10 }\end{array}$} & \multicolumn{7}{|c|}{ Idade (anos)/Mulheres [n = 238] (\%) } & \multicolumn{7}{|c|}{ Idade (anos)/Homens [n = 109] (\%) } \\
\hline & $1-4$ & $5-9$ & $10-14$ & $15-19$ & 20-34 & $35-59$ & $\geq 60$ (5) & $1-4$ & $5-9$ & $10-14$ & $15-19$ & $20-34$ & $35-59$ & $\geq 60$ \\
\hline & {$[n=3]$} & {$[n=1]$} & {$[n=1]$} & {$[n=2]$} & {$[n=14]$} & {$[n=76]$} & {$[n=141]$} & {$[n=13]$} & {$[n=1]$} & {$[n=0]$} & {$[n=3]$} & {$[n=12]$} & {$[n=28]$} & {$[n=52]$} \\
\hline \multicolumn{15}{|l|}{$2000-2002$} \\
\hline $\begin{array}{l}\text { Outras septicemias } \\
\text { (A41) }\end{array}$ & - & - & - & - & $1(7,1)$ & $6(7,9)$ & $20(14,2)$ & $2(15,4)$ & - & - & - & - & $4(14,3)$ & $2(3,8)$ \\
\hline $\begin{array}{l}\text { Diabetes mellitus } \\
\text { (E10-E14) }\end{array}$ & - & - & - & - & - & $1(1,3)$ & $10(7,1)$ & - & - & - & - & - & $1(3,6)$ & $3(5,8)$ \\
\hline Obesidade (E66) & - & - & - & - & - & $2(2,6)$ & $1(0,7)$ & - & - & - & - & - & $1(3,6)$ & - \\
\hline $\begin{array}{l}\text { Doenças hipertensivas } \\
(|10-| 15)\end{array}$ & - & - & - & - & - & $10(13,2)$ & $18(12,8)$ & - & - & - & - & - & $4(14,3)$ & $7(13,5)$ \\
\hline $\begin{array}{l}\text { Doenças isquêmicas } \\
\text { do coração (I20-I25) }\end{array}$ & - & - & - & - & - & $4(5,3)$ & $3(2,1)$ & - & - & - & - & $1(8,3)$ & $1(3,6)$ & $4(7,7)$ \\
\hline $\begin{array}{l}\text { Insuficiência cardíaca } \\
\text { congestiva (I50) }\end{array}$ & - & - & - & - & - & $1(1,3)$ & $8(5,7)$ & - & - & - & - & - & $1(3,6)$ & $1(1.9)$ \\
\hline $\begin{array}{l}\text { Demais doenças } \\
\text { cardiovasculares } \\
(126-144,147-149)\end{array}$ & - & - & - & - & $1(7,1)$ & $4(5,3)$ & $16(11,3)$ & $2(15,4)$ & - & - & - & - & - & $2(3,8)$ \\
\hline $\begin{array}{l}\text { Pneumonias } \\
\text { (J15-J18) }\end{array}$ & $2(66,7)$ & $\begin{array}{c}1 \\
(100,0)\end{array}$ & - & - & $1(7,1)$ & $7(9,2)$ & $18(12,8)$ & $7(53,9)$ & - & - & $2(66,7)$ & - & $2(7,1)$ & $9(17,3)$ \\
\hline DPOC (J40-J44, J47) & - & - & - & - & $1(7,1)$ & $2(2,6)$ & $21(14,9)$ & - & - & - & & $1(8,3)$ & $1(3,6)$ & $9(17,3)$ \\
\hline $\begin{array}{l}\text { Insuficiência respi- } \\
\text { ratória, não classifi- } \\
\text { cada em outra parte } \\
\text { (J96) }\end{array}$ & $2(66,7)$ & $\begin{array}{c}1 \\
(100,0)\end{array}$ & $1(100,0)$ & $2(100,0)$ & $6(42,9)$ & $48(63,2)$ & $83(58,9)$ & $9(69,2)$ & $\begin{array}{c}1 \\
(100,0)\end{array}$ & - & $1(33,3)$ & $10(83,0)$ & $6(21,4)$ & $35(67,3)$ \\
\hline $\begin{array}{l}\text { Outros transtornos } \\
\text { respiratórios (J98) }\end{array}$ & - & - & - & - & $2(14,2)$ & $6(7,9)$ & $18(12,9)$ & - & - & - & - & $1(8,3)$ & $4(14,3)$ & $9(17,3)$ \\
\hline $\begin{array}{l}\text { Parada cardiorrespi- } \\
\text { ratória (R09.2) }\end{array}$ & - & - & - & - & $3(21,3)$ & $4(5,3)$ & $20(14,2)$ & $2(15,4)$ & - & - & - & $1(8,3)$ & $6(21,4)$ & $6(11,5)$ \\
\hline $\begin{array}{l}\text { Outras causas as- } \\
\text { sociadas }\end{array}$ & $1(33,3)$ & - & - & - & $2(14,2)$ & $20(26,3)$ & $53(37,6)$ & $6(46,2)$ & - & - & $\begin{array}{c}3 \\
(100,0)\end{array}$ & $4(33,3)$ & $10(35,7)$ & $19(36,5)$ \\
\hline
\end{tabular}

(continua)

o vírus influenza são fatores que podem prevenir as crises de asma e as internações, contribuindo para a redução da morbimortalidade por asma. A implementação de programas de combate e controle da asma na Argentina, por exemplo, levou à redução das taxas de mortalidade, que foi associada ao uso sistemático do corticoide inalatório no controle da asma e na orientação do paciente quanto à importância da adesão ao tratamento, a partir de 1996 31. No Município do Rio de Janeiro, dados do Ministério da Saúde mostram que as internações pela doença reduziram em 56,3\% no período de 2004-2009, em crianças com até 14 anos de idade (http://www.datasus. gov.br, acessado em 13/Nov/2012). Esse período coincide com a implementação do programa de controle da asma no Município do Rio de Janeiro, o RespiraRio.

Uma limitação deste estudo diz respeito à escolha do SIM como única fonte de dados. As estatísticas de mortalidade são dependentes da qualidade dos registros sobre a causa básica de morte e da precisão dos diagnósticos feitos pelos médicos. A perda de informações produz falhas no conhecimento da mortalidade segundo causas e baixa acurácia. Além disso, as falhas na DO contribuem para a não completude das variáveis sociodemográficas do SIM. Os dados notificados no SIM são os mais fidedignos na avaliação dos eventos de mortes, mas como o preenchimento das DO ainda não é satisfatório, observa-se uma proporção de campos não preenchidos. Apesar 


\begin{tabular}{|c|c|c|c|c|c|c|c|c|c|c|c|c|c|c|}
\hline & \multicolumn{7}{|c|}{ Idade (anos)/Mulheres $(n=232)(\%)$} & \multicolumn{7}{|c|}{ Idade $($ anos $) /$ Homens $(n=73)(\%)$} \\
\hline & $\begin{array}{c}1-4 \\
{[n=4]}\end{array}$ & $\begin{array}{c}5-9 \\
{[n=2]}\end{array}$ & $\begin{array}{c}10-14 \\
{[n=1]}\end{array}$ & $\begin{array}{l}15-19 \\
{[n=3]}\end{array}$ & $\begin{array}{l}20-34 \\
{[n=7]}\end{array}$ & $\begin{array}{c}35-59 \\
{[n=67]}\end{array}$ & $\begin{aligned} & \geq 60 \\
{[n} & =148]\end{aligned}$ & $\begin{array}{c}1-4 \\
{[n=6]}\end{array}$ & $\begin{array}{c}5-9 \\
{[n=0]}\end{array}$ & $\begin{array}{c}10-14 \\
{[n=1]}\end{array}$ & $\begin{array}{l}15-19 \\
{[n=1]}\end{array}$ & $\begin{array}{l}20-34 \\
{[n=3]}\end{array}$ & $\begin{array}{c}35-59 \\
{[n=23]}\end{array}$ & $\begin{array}{c}\geq 60 \\
{[n=39]}\end{array}$ \\
\hline \multicolumn{15}{|l|}{ 2007-2009 } \\
\hline $\begin{array}{l}\text { Outras septicemias } \\
\text { (A41) }\end{array}$ & $2(50,0)$ & $1(50,0)$ & - & $1(33,3)$ & $2(28,6)$ & $4(6,0)$ & $44(29,7)$ & $1(16,7)$ & - & $1(100,0)$ & - & - & $4(17,4)$ & $4(10,3)$ \\
\hline $\begin{array}{l}\text { Diabetes mellitus } \\
\text { (E10-E14) }\end{array}$ & - & - & - & $1(33,3)$ & - & $3(4,5)$ & $13(8,8)$ & - & - & - & - & - & - & $4(10,3)$ \\
\hline Obesidade (E66) & - & - & - & - & $1(14,3)$ & $3(4,5)$ & $1(0,7)$ & - & - & - & - & - & - & - \\
\hline $\begin{array}{l}\text { Doenças hipertensivas } \\
\text { (I10-|15) }\end{array}$ & - & - & - & - & $1(14,3)$ & $16(23,8)$ & $35(23,6)$ & - & - & - & - & $1(33,3)$ & $4(17,4)$ & $7(17,9)$ \\
\hline $\begin{array}{l}\text { Doenças isquêmicas } \\
\text { do coração (120-125) }\end{array}$ & - & - & - & - & - & $2(3,0)$ & $14(9,5)$ & - & - & - & - & - & $3(13,0)$ & $5(12,8)$ \\
\hline $\begin{array}{l}\text { Insuficiência cardíaca } \\
\text { congestiva (I50) }\end{array}$ & - & - & - & - & - & $2(3,0)$ & $9(6,1)$ & - & - & - & - & - & - & - \\
\hline $\begin{array}{l}\text { Demais doenças } \\
\text { cardiovasculares } \\
(126-144,147-149)\end{array}$ & - & - & - & - & $1(14,3)$ & $6(9,0)$ & $16(10,8)$ & - & - & - & - & - & - & $1(2,6)$ \\
\hline $\begin{array}{l}\text { Pneumonias } \\
\text { (J15-J18) }\end{array}$ & $2(50,0)$ & $1(50,0)$ & $1(100,0)$ & $1(33,3)$ & $3(42,9)$ & $10(14,9)$ & $57(38,5)$ & $2(33,3)$ & - & - & $\begin{array}{c}1 \\
(100,0)\end{array}$ & - & $6(26,1)$ & $8(20,5)$ \\
\hline DPOC (J40-J44, J47) & - & - & - & - & - & $6(9,0)$ & $16(10,8)$ & - & - & - & - & - & $2(8,7)$ & $5(12,8)$ \\
\hline $\begin{array}{l}\text { Insuficiência respi- } \\
\text { ratória, não classifi- } \\
\text { cada em outra } \\
\text { parte (J96) }\end{array}$ & $2(50,0)$ & $1(50,0)$ & - & $1(33,3)$ & $5(71,4)$ & $48(71,6)$ & $76(51,4)$ & $2(33,3)$ & - & - & $\begin{array}{c}1 \\
(100,0)\end{array}$ & - & $11(47,8)$ & ) $16(41,0)$ \\
\hline $\begin{array}{l}\text { Outros transtornos } \\
\text { respiratórios (J98) }\end{array}$ & - & - & - & - & - & $3(4,5)$ & $15(10,1)$ & $1(16,7)$ & - & $1(100,0)$ & $\begin{array}{c}1 \\
(100,0)\end{array}$ & - & $3(13,0)$ & $3(7,7)$ \\
\hline $\begin{array}{l}\text { Parada cardiorrespi- } \\
\text { ratória (R09.2) }\end{array}$ & - & - & - & - & - & $6(9,0)$ & $12(8,1)$ & - & - & - & - & - & $5(21,7)$ & $4(10,3)$ \\
\hline $\begin{array}{l}\text { Outras causas } \\
\text { associadas }\end{array}$ & $3(75,0)$ & $1(50,0)$ & - & $1(33,3)$ & $4(57,1)$ & $27(40,3)$ & $77(52,0)$ & $\begin{array}{c}6 \\
(100,0)\end{array}$ & - & - & - & $1(33,3)$ & $6(26,1)$ & $22(56,4)$ \\
\hline
\end{tabular}

CID-10: 10a revisão da Classificação Internacional de Doenças; DPOC: Doença pulmonar obstrutiva crônica.

Fonte: Departamento de Informática do SUS (Sistema de Informações sobre Mortalidade. http://www.datasus.gov.br, acessado em 05/Out/2011).

da expressiva melhora na qualidade da informação dos dados do SIM, optou-se, neste trabalho, pela não inclusão das variáveis raça/cor e escolaridade, postergando para futuras investigações o estudo da influência destas variáveis na morbimortalidade por asma.

Outros fatores podem comprometer a validade e a acurácia das informações contidas na DO, como mudanças nas classificações das doenças e desconhecimento do diagnóstico pelo médico, que levam ao aumento na frequência de registros de óbitos falsos positivos e falsos negativos para o diagnóstico de asma, podendo resultar em superestimação ou subestimação da mortalidade por asma. A identificação desses óbitos requer estudos de validação, com análise de todas as mortes que mencionaram asma, e daquelas que citaram as comorbidades mais associadas à asma, além da obtenção de informações sobre o histórico da doença, detalhes das circunstâncias do óbito e até resultados de autópsia. A fidedignidade dos óbitos por asma tem sido investigada por vários autores, os quais mostram que estes óbitos têm baixa sensibilidade e alta especificidade. Hunt et al. 20 analisaram 339 DO de uma coorte de asmáticos entre 1964 e 1983 e encontraram 22 menções de asma. Após a investigação desses óbitos, os especialistas classificaram a asma como causa básica em 53 óbitos, o que corresponde a uma sensibilidade da DO de $42 \%$. Entre os demais 286 óbitos, apenas quatro DO mencionaram a asma, representando uma especificidade de $99 \%$.

A presença de comorbidades e complicações no momento do óbito está associada a um maior número de registros de causas na $\mathrm{DO}$. O número médio de causas informadas nas DO é um dos 
Causas básicas quando a asma é referida como causa associada na Declaração de Óbito entre óbitos, segundo gênero e faixa etária. Município do Rio de Janeiro, Brasil, 2000-2002 e 2007-2009.

\begin{tabular}{|c|c|c|c|c|c|c|c|c|c|c|c|c|c|c|}
\hline \multirow[t]{3}{*}{ Causas básicas (CID-10) } & \multicolumn{7}{|c|}{ Idade (anos)/Mulheres $[n=122]$} & \multicolumn{7}{|c|}{ Idade (anos)/Homens $[\mathrm{n}=58]$} \\
\hline & $1-4$ & $5-9$ & $10-14$ & $15-19$ & $20-34$ & $35-59$ & $\geq 60$ & $1-4$ & $5-9$ & $10-14$ & $15-19$ & $20-34$ & $35-59$ & $\geq 60$ \\
\hline & $\mathrm{n}$ & $\mathrm{n}$ & $\mathbf{n}$ & $\mathbf{n}$ & $\mathbf{n}$ & $\mathbf{n}$ & $\mathrm{n}$ & $\mathrm{n}$ & $\mathrm{n}$ & $\mathrm{n}$ & $\mathbf{n}$ & $\mathrm{n}$ & $\mathrm{n}$ & $\mathrm{n}$ \\
\hline \multicolumn{15}{|l|}{ 2000-2002 } \\
\hline $\begin{array}{l}\text { Algumas doenças infec- } \\
\text { ciosas e parasitárias } \\
\text { (A00-B99) }\end{array}$ & - & - & - & - & - & $3(12,0)$ & $3(3,3)$ & - & - & - & - & - & $2(16,7)$ & $1(2,4)$ \\
\hline $\begin{array}{l}\text { Neoplasia de pulmão e } \\
\text { pleura (C34) }\end{array}$ & - & - & - & - & - & - & $2(2,2)$ & - & - & $1(50,0)$ & - & - & - & - \\
\hline $\begin{array}{l}\text { Neoplasia (C00-C33, } \\
\text { C34-D48) }\end{array}$ & - & - & - & - & - & $2(8,0)$ & $6(6,6)$ & - & - & - & - & - & - & $4(9,5)$ \\
\hline $\begin{array}{l}\text { Diabetes mellitus } \\
\text { (E10-E14) }\end{array}$ & - & - & - & - & - & $3(12,0)$ & $6(6,6)$ & - & - & - & - & - & - & $2(4,8)$ \\
\hline $\begin{array}{l}\text { Doenças hipertensivas } \\
(|10-| 15)\end{array}$ & - & - & - & - & - & $4(16,0)$ & $8(8,8)$ & - & - & - & - & - & - & $2(4,8)$ \\
\hline $\begin{array}{l}\text { Doenças isquêmicas do } \\
\text { coração (I20-125) }\end{array}$ & - & - & - & - & - & $2(8,0)$ & $\begin{array}{c}13 \\
(14,3)\end{array}$ & - & - & - & - & - & $3(25,0)$ & $9(21,4)$ \\
\hline $\begin{array}{l}\text { Insuficiência cardíaca } \\
\text { congestiva (I50) }\end{array}$ & - & - & - & - & - & $1(4,0)$ & $2(2,2)$ & - & - & - & - & - & $1(8,3)$ & $3(7,1)$ \\
\hline $\begin{array}{l}\text { Demais doenças do } \\
\text { aparelho circulatório } \\
(126-142,148-149,151)\end{array}$ & - & - & - & - & - & $1(4,0)$ & $4(4,4)$ & - & - & - & - & - & - & $3(7,1)$ \\
\hline DPOC (J43-J44, J47) & - & - & - & - & - & $3(12,0)$ & $\begin{array}{c}14 \\
(15,4)\end{array}$ & - & - & - & - & - & $3(25,0)$ & $8(19,0)$ \\
\hline $\begin{array}{l}\text { Outros transtornos } \\
\text { respiratórios (J98) }\end{array}$ & - & - & - & - & - & $2(8,0)$ & $8(8,8)$ & - & - & - & - & $1(50,0)$ & - & $4(9,5)$ \\
\hline $\begin{array}{l}\text { Demais doenças do } \\
\text { aparelho respiratório } \\
\text { (J00-J42, J84-J94) }\end{array}$ & - & - & - & - & $1(25,0)$ & $1(4,0)$ & $2(2,2)$ & - & - & - & - & $1(50,0)$ & $2(16,7)$ & $1(2,4)$ \\
\hline $\begin{array}{l}\text { Doenças do aparelho } \\
\text { digestivo }\end{array}$ & - & - & - & - & - & - & $6(6,6)$ & - & - & $1(50,0)$ & - & - & - & $2(4,8)$ \\
\hline Outras causas básicas & & $\begin{array}{c}1 \\
(100,0)\end{array}$ & - & $\begin{array}{c}1 \\
(100,0)\end{array}$ & $3(75,0)$ & $3(12,0)$ & $\begin{array}{c}17 \\
(18,7)\end{array}$ & & & & & - & $1(8,3)$ & $3(7,1)$ \\
\hline Total (\%) & - & $\begin{array}{c}1 \\
(100,0)\end{array}$ & - & $\begin{array}{c}1 \\
(100,0)\end{array}$ & $\begin{array}{c}4 \\
(100,0)\end{array}$ & $\begin{array}{c}25 \\
(100,0)\end{array}$ & $\begin{array}{c}91 \\
(100,0)\end{array}$ & - & - & $2(100,0)$ & - & $\begin{array}{c}2 \\
(100,0)\end{array}$ & $\begin{array}{c}12 \\
(100,0)\end{array}$ & $42(100,0)$ \\
\hline
\end{tabular}

(continua)

principais indicadores relacionados à metodologia de causas múltiplas. No presente estudo, o número médio de causas mencionadas por DO, considerando causas múltiplas, foi equivalente a 3,4, sendo este superior à média do Brasil em 2003, que foi de 2,81 para as doenças em geral 32 . Neste estudo, $96 \%$ dos óbitos apresentaram mais de uma causa na DO, o que significa que haveria perda de informação se apenas a causa básica fosse analisada. Nos Estados Unidos, em 1917 apenas $35 \%$ dos óbitos tinham mais de uma causa descrita na DO; em 1955 passou a 60\%, e em
1979 uma proporção de 75\% dos óbitos registravam mais de uma causa por óbito 33 .

Ao avaliar as causas mencionadas na DO, quando a asma foi classificada como causa básica no primeiro e último triênios, observou-se que as doenças mais assinaladas eram as do aparelho respiratório, seguidas das doenças do aparelho circulatório. As causas associadas mais frequentes foram a insuficiência respiratória, a pneumonia, a septicemia e a hipertensão arterial em ambos os triênios. Esses resultados são consistentes com os dados apresentados por Omachi et 


\begin{tabular}{|c|c|c|c|c|c|c|c|c|c|c|c|c|c|c|}
\hline \multirow[t]{3}{*}{ Causas básicas (CID-10) } & \multicolumn{7}{|c|}{ Idade (anos)/Mulheres $[\mathrm{n}=118](\%)$} & \multicolumn{7}{|c|}{ Idade (anos)/Homens [n = 36] (\%) } \\
\hline & $1-4$ & $5-9$ & $10-14$ & $15-19$ & $20-34$ & $35-59$ & $\geq 60$ & $1-4$ & $5-9$ & 10-14 & $15-19$ & $20-34$ & $35-59$ & $\geq 60$ \\
\hline & $n$ & $\mathrm{n}$ & $\mathrm{n}$ & $\mathrm{n}$ & $\mathrm{n}$ & $\mathrm{n}$ & $\mathrm{n}$ & $\mathrm{n}$ & $\mathrm{n}$ & $\mathrm{n}$ & $\mathrm{n}$ & $\mathrm{n}$ & $\mathrm{n}$ & $\mathrm{n}$ \\
\hline \multicolumn{15}{|l|}{ 2007-2009 } \\
\hline $\begin{array}{l}\text { Doenças isquêmicas do } \\
\text { coração (I20-125) }\end{array}$ & - & - & - & - & - & $1(3,6)$ & $\begin{array}{c}11 \\
(13,2)\end{array}$ & - & - & - & - & - & $2(40,0)$ & $2(7,7)$ \\
\hline $\begin{array}{l}\text { Insuficiência cardíaca } \\
\text { congestiva (150) }\end{array}$ & - & - & - & - & - & - & $4(4,8)$ & - & - & - & - & - & - & - \\
\hline $\begin{array}{l}\text { Demais doenças do } \\
\text { apare-lho circulatório } \\
(126-142,148-149,151)\end{array}$ & - & - & - & - & - & $1(3,6)$ & $7(8,4)$ & - & - & - & - & - & - & - \\
\hline DPOC (J43-J44, J47) & - & - & - & - & - & $2(7,1)$ & $4(4,8)$ & - & - & - & - & - & - & $5(19,2)$ \\
\hline $\begin{array}{l}\text { Outros transtornos } \\
\text { respiratórios (J98) }\end{array}$ & - & - & - & - & - & - & $1(1,2)$ & - & - & - & - & - & - & - \\
\hline $\begin{array}{l}\text { Demais doenças do } \\
\text { apare-lho respiratório } \\
\text { (J00-J42, J84-J94) }\end{array}$ & - & - & - & - & - & $1(3,6)$ & $3(3,6)$ & - & - & - & - & $2(66,6)$ & - & - \\
\hline $\begin{array}{l}\text { Doenças do aparelho } \\
\text { digestivo }\end{array}$ & - & - & - & - & - & $2(7,1)$ & $6(7,2)$ & - & - & - & - & - & - & $1(3,8)$ \\
\hline Outras causas básicas & - & - & - & - & $4(66,7)$ & $7(25,0)$ & $7(8,4)$ & $\begin{array}{c}1 \\
(100,0)\end{array}$ & - & - & $1(100,0)$ & $1(33,3)$ & $1(20,0)$ & $6(23,1)$ \\
\hline Total (\%) & - & $1(100,0)$ & - & - & $\begin{array}{c}6 \\
(100,0)\end{array}$ & $\begin{array}{c}28 \\
(100,0)\end{array}$ & $\begin{array}{c}83 \\
(100,0)\end{array}$ & $\begin{array}{c}1 \\
(100,0)\end{array}$ & - & - & $1(100,0)$ & $\begin{array}{c}3 \\
(100,00)\end{array}$ & $5(100,0)$ & $\begin{array}{c}26 \\
(100,0)\end{array}$ \\
\hline
\end{tabular}

CID-10: 10ạ revisão da Classificação Internacional de Doenças; DPOC: Doença pulmonar obstrutiva crônica.

Fonte: Departamento de Informática do SUS (Sistema de Informações sobre Mortalidade. http://www.datasus.gov.br, acessado em 05/Out/2011).

al. ${ }^{34}$, que analisaram os óbitos de uma coorte de asmáticos nos Estados Unidos, acompanhados em média por dois anos. Eles verificaram que as doenças respiratórias (63\%) foram as principais causas declaradas nas DO como causas básicas ou associadas 34. No Brasil, Santo 18, em 2000, observou que $58,5 \%$ das causas associadas aos óbitos por asma correspondiam à insuficiência respiratória, 24,3\% à parada cardiorrespiratória, $15,1 \%$ à pneumonia e 9,4\% à doença pulmonar obstrutiva crônica. Chama a atenção, a elevada proporção de menção de insuficiência respiratória e parada cardiorrespiratória nos óbitos por asma, o que talvez se justifique pela gravidade das crises, com alto risco de morte.

É necessário destacar que, apesar de o estudo de associações de causas ser importante na análise por causas múltiplas, a mensuração destas associações ainda não foi padronizada, e vários estudos utilizaram diferentes metodologias. Israel et al. 33 enfatizaram a complexidade do estudo de associação de causas na análise de causas múltiplas, apontando a necessidade de pesquisas adicionais.

Uma questão a ser discutida é a dificuldade em distinguir a asma da DPOC ou a coexistência destas patologias, resultando em falha no regis- tro da causa básica dos óbitos e, consequentemente, na acurácia da informação. Guite \& Burney 35 , em estudo de óbitos ocorridos em coorte com 2.382 pacientes previamente hospitalizados por asma, verificaram que $8 \%$ dos óbitos foram classificados erroneamente, tendo a DPOC como causa básica de morte. No presente trabalho, a DPOC foi a causa associada em 9,8\% dos óbitos, com maior proporção entre homens de 60 anos e mais.

Outro aspecto a ser considerado é a frequente associação entre doenças cardiovasculares e asma. De fato, neste estudo, as doenças do aparelho cardiovascular foram as principais causas básicas, em algumas faixas etárias, dos óbitos em que a asma foi classificada como causa associada, em ambos os triênios estudados. Contudo, tal associação ainda não foi bem estabelecida. Cazzola et al. 36, em inquérito populacional, verificaram que, apesar de as doenças cardiovasculares serem mais prevalentes em pacientes asmáticos do que na população geral, a associação encontrada foi fraca, equivalente a 0,98 (intervalo de $95 \%$ de confiança-IC95\%: 0,90-1,07).

O declínio da mortalidade por asma verificado no final dos anos 90 em vários países foi associado ao uso padronizado dos corticoides 
inalatórios no tratamento de manutenção da asma, como evidenciado em estudo de metanálise que mostrou ter o corticoide inalatório reduzido em aproximadamente $55 \%$ as exacerbações de asma, comparado ao placebo (risco relativo = 0,46; IC95\%) 37. Nesse contexto, a implementação em larga escala de programas de educação em asma, que incluam a distribuição gratuita de medicação de manutenção do controle da doença à população carente, poderia ter forte impacto na redução das taxas de mortalidade por asma.

Os resultados obtidos nesta pesquisa estão na contramão do observado na mortalidade geral no município, que mostrou um predomínio de óbitos entre os homens. Mais estudos são necessários para esclarecer as diferenças entre os sexos na mortalidade por asma, além de séries temporais maiores que possibilitem uma avaliação melhor da evolução das taxas de mortalidade entre as mulheres.

Como conclusão, os achados deste trabalho mostram que a análise por causas múltiplas possibilita a identificação das doenças que participam do processo mórbido que leva à morte por asma e que, sob o enfoque apenas da causa básica, permaneceriam desconhecidas. Ressalta-se que o emprego da metodologia das causas múltiplas não substitui o estudo da causa básica. Devido à importante subestimação observada neste estudo, a utilização dessa metodologia deveria ser considerada nas estatísticas de mortalidade da asma, visando ao conhecimento da real dimensão de ocorrência da doença e auxiliando na implementação de programas de prevenção e tratamento específicos. Evidenciam, também, o consistente declínio da mortalidade por asma entre homens, que não foi acompanhado pelas mulheres, e a necessidade de melhor compreender este processo. As razões que implicam diferenças segundo gênero na etiologia e na evolução clínica da asma merecem ser mais investigadas. Outros estudos devem ser conduzidos a fim de preencher tais lacunas e contribuir de forma efetiva para o controle da asma no país.

Com base nas conclusões obtidas neste trabalho, algumas recomendações podem ser especificadas: utilização da metodologia das causas múltiplas rotineiramente nas estatísticas de mortalidade por asma, expansão da cobertura dos programas de combate à asma e orientação aos médicos quanto ao preenchimento correto da DO.

\section{Resumen}

El estudio de las causas múltiples de óbitos permite conocer la extensión real de las estadísticas de mortalidad. El objetivo de esta investigación fue evaluar la tendencia de las tasas de mortalidad, relacionada con el asma, en el municipio de Río de Janeiro, Brasil, en el período de 2000-2009, con los datos obtenidos en el Sistema de Información de Mortalidad (SIM), de individuos con un año o más de edad, en quienes el asma fue mencionada en cualquier línea o parte del certificado médico de defunción. Para el análisis de datos fue utilizada la técnica de regresión lineal. La serie histórica mostró una tendencia al declive en las tasas de mortalidad estandarizadas, relacionada con el as ma como causa básica y múltiple, con reducción entre los hombres y estabilidad entre las mujeres. Cuando el asma fue la causa básica, las razones asociadas más frecuentes fueron enfermedades del aparato respiratorio. La mortalidad relacionada con el asma fue subestimada cuando se consideraba solamente como causa básica, lo que podría ser evitado con la utilización de la metodología de causas múltiples en las estadísticas de mortalidad por asma.

Asma; Causas Múltiples de Muerte; Mortalidad 


\section{Colaboradores}

E. M. Silva contribuiu na concepção, planejamento e execução do trabalho científico, interpretação dos resultados e escrita do manuscrito. G. Azevedo e Silva colaborou na concepção, planejamento, interpretação dos resultados e aprovação final da versão a ser publicada.

\section{Referências}

1. Masoli M, Fabian D, Holt S, Beasley R, for Asthma GINA Program GI. The global burden of asthma: executive summary of the GINA Dissemination Committee Report. Allergy 2004; 59:469-78.

2. Wijesinghe M, Weatherall M, Perrin K, Crane J, Beasley R. International trends in asthma mortality rates in the 5- to 34-year age group: a call for closer surveillance. Chest 2009; 135:1045-9.

3. Campos HS. Mortalidade por asma no Brasil, 19801998. Pulmão RJ 2003; 12:208-16.

4. Solé D, Melo KC, Camelo-Nunes IC, Freitas LS, Britto M, Rosário NA, et al. Changes in the prevalence of asthma and allergic diseases among Brazilian schoolchildren (13-14 years old): comparison between ISAAC phases one and three. J Trop Pediatr 2007; 53:13-2.

5. Silverman RA, Boudreaux ED, Woodruff PG, Clark $\mathrm{S}$, Camargo CA. Cigarette smoking among asthmatic adults presenting to 64 emergency departments. Chest 2003; 123:1472-9.

6. Chaudhuri R, Livingston E, McMahon AD, Lafferty J, Fraser I, Spears M, et al. Effects of smoking cessation on lung function and airway inflammation in smokers with asthma. Am J Respir Crit Care Med 2006; 174:127-33.

7. Secretaria de Vigilância em Saúde, Secretaria de Gestão Estratégica e Participativa, Ministério da Saúde. VIGITEL Brasil 2009: vigilância de fatores de risco e proteção para doenças crônicas por inquérito telefônico. Brasília: Ministério da Saúde; 2010.
8. Kumana CR, Kou M, Lauder IJ, Ip MS, Lam WK. Increasing use of inhaled steroids associated with declining asthma mortality. J Asthma 2001; 38:161-7.

9. Suissa S. Inhaled corticosteroids: impact on asthma morbidity and mortality. J Allergy Clin Immunol 2001; 107:937-44.

10. Wenderson CCA, Lasmar MLBF, Camargos PAM. Asma aguda na infância: o impacto do uso do corticóide inalatório e fatores associados às hospitalizações e consultas de urgência. Rev Méd Minas Gerais 2008; 18(4 Suppl 3):S34-9.

11. Camargos PAM, Profeta SC. Tratamento de manutenção da asma persistente à admissão em ambulatório de pneumologia pediátrica. J Pediatr (Rio J.) 2003; 79:233-8.

12. Cerci Neto A, Ferreira Filho OF, Bueno T, Talhari MA. Redução do número de internações hospitalares por asma após a implantação de programa multiprofissional de controle da asma na cidade de Londrina. J Bras Pneumol 2008; 34:639-45.

13. Souza-Machado C, Souza-Machado A, Franco R, Ponte EV, Cruz AA. Impacto do PROAR (programa para o controle da asma e da rinite alérgica na Bahia) sobre a utilização de recursos de saúde, custos e morbi-mortalidade por asma em Salvador. Gazeta Médica da Bahia 2008; (78):59-63.

14. Souza-Machado C, Souza-Machado A, Cruz AA. Asthma mortality inequalities in Brazil: tolerating the unbearable. ScientificWorldJournal 2012; 2012:625829. 
15. Cerci Neto A, Ferreira Filho OF, Bueno T. Exemplos brasileiros de programas de controle de asma. Bras Pneumol 2008; 34:103-6.

16. Valle SOR, Guimarães MHFS, Scorza EC, Amancio JO, Freitas N, Silva MAV. Plano de atenção ao paciente com asma e rinite - RespirARio: análise de 5 anos. In: Congresso Brasileiro de Epidemiologia. http://www.epi2011.com.br (acessado em 13/ Nov/2012).

17. Furhrman C, Jougla E, Uhry Z, Delmas MC. Deaths with asthma in France, 2000-2005: a multiplecause analysis. J Asthma 2009; 46:402-6.

18. Santo AH. Mortalidade relacionada à asma, Brasil, 2000: um estudo usando causas múltiplas de morte. Cad Saúde Pública 2006; 22:41-52.

19. Santo AH, Pinheiro CE. Causas múltiplas de morte relacionadas à asma, Rio Grande do Sul e São Paulo, 1999 e 2000. J Pneumol 2003; 29 Suppl 1:S33.

20. Hunt Jr. LW, Silverstein MD, Reed CE, O'Connell EJ, O'Fallon WM, Yunginger JW. Accuracy of the death certificate in a population-based study of asthmatic patients. JAMA 1993; 269:1947-52.

21. Gould WW. Linear splines and piecewise linear functions. Stata Technical Bulletin 1993; 15:13-7.

22. Moorman JE, Rudd RAA, Johnson CA, King M, Minor P, Bailey C, et al. National surveillance for asthma-United States, 1980-2004. MMWR Surveill Summ 2002; 51:1-13.

23. Oliveira LSC, Machado JL, Duarte PAD, Tondo LG Jorge MM, Marchiori R, et al. Taxa de mortalidade por asma de acordo com os atestados de óbito nos moradores em Cascavel/PR no período de jan/05 a dez/09. Pulmão RJ 2010; 19:8-12

24. Leynaert B, Bousquet J, Henry C, Liard R, Neukirch F. Is bronchial hyperresponsiveness more frequent in women than in men? A population-based study. Am J Respir Crit Care Med 1997; 156:1413-20.

25. Goodman DE, Israel E, Rosenberg M, Johnston R, Weiss ST, Drazen JM. The influence of age, diagnosis, and gender on proper use of metered-dose inhalers. Am J Respir Crit Care Med 1994; 150(5 Pt 1):1256-61.

26. McLeish AC, Zvolensky MJ. Asthma and cigarette smoking: a review of the empirical literature. Asthma 2010; 47:345-61.
27. McCoy L, Redelings M, Sorvillo F, Simon P. A mul tiple cause-of-death analysis of asthma mortality in the United States, 1990-2001. J Asthma 2005; 42:757-63.

28. Chatkin G, Chatkin JMM, Fritscher CCC, CavaletBlanco D, Bittencourt HRR, Sears MR. Asthma mortality in southern Brazil: is there a changing trend? J Asthma 2007; 44:133-6.

29. Azevedo e Silva G, Valente, JG, Malta DC. Tendências do tabagismo na população adulta das capitais brasileiras: uma análise dos dados de inquéritos telefônicos de 2006 a 2009. Rev Bras Epidemiol 2011; 14:103-14.

30. Reid DWEC, Hendrick VJ, Aitken TC, Berrill WT, Stenton SC, Hendrick DJ. Age-dependent inaccuracy of asthma death certification in Northern England, 1991-1992. Eur Respir J 1998; 12:1079-83.

31. Neffen H, Baenacagnani C, Passalacqua G, Canonica G, Rocco D. Asthma mortality, inhaled steroids, and changing asthma therapy in Argentina (19901999). Respir Med 2006; 100:1431-5.

32. Santo AH. Potencial epidemiológico da utilização das causas múltiplas de morte por meio de suas menções nas declarações de óbito, Brasil, 2003. Rev Panam Salud Pública 2007; 22:178-86.

33. Israel RA, Rosenberg HM, Curtin LR. Analytical potential for multilple cause-of-death data. Am J Epidemiol 1986; 124:161-79.

34. Omachi TA, Iribarren C, Sarkar U, Tolstykh I, Yelin $\mathrm{EH}$, Katz PP, et al. Risk factors for death in adults with severe asthma. Ann Allergy Asthma Immunol 2008; 101:130-6.

35. Guite HF, Burney PG. Accuracy of recording of deaths from asthma in the UK: the false negative rate. Thorax 1996; 51:924-8.

36. Cazzola M, Calzetta L, Bettoncelli G, Cricelli C, Romeo F, Matera MGG, et al. Cardiovascular disease in asthma and COPD: a population-based retro spective cross-sectional study. Respir Med 2012; 106:249-56.

37. Sin DD, Man J, Sharpe H, Gan WQQ, Man PF. Pharmacological management to reduce exacerbations in adults with asthma: a systematic review and meta-analysis. JAMA 2004; 292:367-76

Recebido em 21/Ago/2012

Versão final reapresentada em 15/Nov/2012

Aprovado em 03/Dez/2012 